\title{
SOBRE LA PROMESA DE UNA FENOMENOLOGÍA ASUBJETIVA ${ }^{1}$
}

James Dodd

New School for Social Research, Nueva York, USA

Resumen: Este ensayo describe algunos de los gestos filosóficos clave que definen el proyecto de Jan Patočka de una "fenomenología asubjetiva", con especial atención a los temas del mundo, el sentido, el cuerpo, el movimiento, la individualidad, la reflexión y la crítica al cartesianismo. El ensayo concluye con algunas observaciones sobre los recursos en el pensamiento de Patočka para una reflexión filosófica sobre la animalidad.

Descriptores: Fenomenología $\cdot$ A priori $\cdot$ Mundo $\cdot$ Cuerpo $\cdot$ Reflexión $\cdot$ Animalidad $\cdot$ Individualidad $\cdot$ Cartesianismo

Abstract: This essay outlines some of the key philosophical gestures that define Jan Patočka's project of an "asubjective phenomenology," with particular attention paid to the themes of the world, meaning, body, movement, selfhood, reflection, and the critique of Cartesianism. The essay concludes with some remarks on the resources in Patocka's thinking for a philosophical reflection on animality.

Keywords: Phenomenology $\cdot$ A priori $\cdot$ World $\cdot$ Body $\cdot$ Reflection $\cdot$ Animality $\cdot$ Selfhood Cartesi-anism

Uno de los capítulos centrales en el estudio del pensamiento de Jan Patočka es el proyecto de lo que él llamó una "fenomenología asubjetiva" el cual comenzó aproximadamente a mediados de la década de 1960 y continuó hasta su muerte en 1977. Este proyecto se basó en tres décadas de reflexión sobre las obras de Edmund Husserl y Martin Heidegger, y tenía como objetivo nada menos que una revisión fundamental de la fenomenología clásica. Mi objetivo en lo que sigue es intentar expresar algo del espíritu -o la promesa- del proyecto de Patočka, a través de una serie de comentarios sobre las ideas y motivaciones que lo animaron.

\footnotetext{
${ }^{1}$ Este texto ha sido traducido por Jorge Enrique Pulido Blanco.

${ }^{2}$ Patočka (1970), y Patočka (1971).
} 


\section{LO A-SUBJETIVO NO ES NO-SUBJETIVO}

Mi primer comentario es que la expresión fenomenología $a$-subjetiva no es equivalente a fenomenología no-subjetiva. Los argumentos en contra del subjetivismo no implican necesariamente un rechazo del concepto del sujeto, ni siquiera implican negarle al sujeto un papel central, incluso constitutivo. Este es el caso de Patočka: "asubjetivo" tiene la intención de designar cómo se vería la fenomenología clásica una vez que se haya liberado de los efectos distorsionantes del subjetivismo, y eso incluye establecer concepciones alternativas del ego, de la individualidad y del "sujeto" pertenecientes al campo fenomenológico cuando se lo articula de nuevo.

Esto es quizás más evidente en la forma en que Patočka aborda el complejo tema del mundo. Por un lado, Patočka se esfuerza por liberar este tema de su reducción a la vida trascendental, pues consideraba que Husserl la identificó erróneamente como el "origen" del sentido del mundo. ${ }^{3}$ Husserl había argumentado que nuestro sentido del mundo se encuentra en la estructura inmanente de la misma vida consciente como una auto-temporalización, como un flujo absoluto. Patočka se opone enérgicamente a esta concepción, pero, sin perder, por otro lado, el control de una perspectiva central que, sin embargo, está incorporada en ella: el papel central de la subjetividad en cualquier descripción del carácter específicamente horizontal del mundo.

La manera en que Patočka entiende este papel de la subjetividad se puede ilustrar con una mirada al ensayo de 1976 El mundo "natural" y la fenomenología, en el que describe dos "polos" constitutivos del mundo natural. El primero es una periferia que circunscribe el alcance externo de las cosas y la espacialidad en la que están articuladas; el segundo es una interioridad subjetiva, en tensión con la periferia, que le imprime a la espacialidad del mundo la forma de una perspectiva (Patočka, 1967, pp. 250-251). Gracias a la tensión que se articula en estos dos polos, la espacialidad del mundo se despliega a medida que el movimiento de la vida personal avanza hacia el exterior, cuya subjetividad interna es tan fundamental como la distancia de la otredad marcada por la periferia.

Sin embargo, las descripciones de esta estructura solo son viables si nos resistimos a entender la "interioridad" del sujeto en la línea de una concepción cartesiana de la inmanencia, donde asumiría el carácter de una región privilegiada de la existencia. Para Patočka, el subjetivismo de Husserl se reduce a la afirmación de que la relación entre sujeto y mundo puede entenderse totalmente en términos de lo que está contenido y asegurado en la inmanencia subjetiva (Patočka, 1972, p. 233). No obstante, la crítica aquí no debe apuntar al re-establecimiento de

\footnotetext{
${ }^{3}$ Véase: Husserl (1950), §11.
} 
un realismo ingenuo, según el cual el privilegio de la subjetividad cedería a la afirmación de que no hay diferencia en el ser que distinga la existencia subjetiva de la no subjetiva. La idea de una fenomenología asubjetiva se orienta, por su parte, en torno a la descripción de una tensión entre interioridad y periferia, individualidad y horizonte, de tal manera que evade las confusiones tanto del cartesianismo como del realismo dogmático.

\section{EL PROBLEMA DEL SENTIDO}

Mi segunda observación es que el enfoque de Patočka desafía hasta qué punto se puede decir que la teoría husserlinana de la intencionalidad define el problema del sentido. A diferencia de Husserl, los logros del comprender, o la forma en que el sentido proporciona orientación, ya no son identificados por Patočka con actos intencionales que tienen como contenido principal una presencia articulada, $o$ una unidad de sentido postulada, si por eso entendemos algo completo y acabado. Por su parte, en las descripciones de Patočka, el campo del sentido y el tipo de "totalidad" que representa surgen como algo determinado por la dinámica de un comprender que es, en el fondo, más un enigma para sí mismo que un orden de convicciones acabado, especialmente cuando se trata de la cuestión de los orígenes.

Es este enfoque del campo del sentido el que está detrás, a mi juicio, del interés que mostró Patočka durante toda su vida por fenómenos como los mitos, las obras de literatura y el lenguaje mismo en cuanto temas para la interrogación filosófica. Aquí podemos señalar la estrategia retórica al principio del ensayo de 1969 ¿Qué es la existencia?, el cual comienza debatiendo con una selección de personajes literarios de las novelas de William Faulkner, Thomas Mann y Fyodor Dostoyevski (Patočka, 1969). Uno no encuentra aquí un "análisis intencional”, al menos no en la forma en que se aborda la unidad intencional de un conjunto de hábitos y creencias acabadas, completas, dadas a la conciencia que se presta a la explicación. Existe, en cambio, un compromiso con el mundo no-unívoco de las expresiones literarias (como el retrato de Adrian Leverkuhn de Doktor Faustus de Thomas Mann), las cuales se consideran representaciones que iluminan la existencia humana en formas que tal vez comprendemos, pero que difícilmente se aprovechan dentro de un análisis sistemático. En tales ejercicios, la reflexión filosófica todavía implica un intento de convertir el "sentido" en un objeto, o al menos en un tema; solamente que lo que cuenta aquí no son solo aquellos casos en los que el sentido es algo cerrado y fijo, un resultado que se puede comprender en términos de univocidad dada o incluso anticipada. El sentido aquí es, de acuerdo con lo anterior, algo más parecido a una pregunta continuamente enriquecida que a una posición, o una creencia que se sometería a un análisis de resultados parciales y a justificaciones. 


\section{EL A PRIORI DEL MUNDO.}

De esta manera, el sentido, que tiene en tal intencionalidad su vida, es descrito por Patočka no solo como algo incrustado en el horizonte de la existencia, sino además como algo horizontal en su esencia básica (Patočka, 1967, p. 253). Más aún, este carácter horizontal del sentido pertenece, para Patočka, al a priori del mundo, y en una forma tal que, como lo expresa en un texto inédito de principios de la década de 1970, contrasta fuertemente con la concepción husserliana del $a$ priori como esencia, como un eidos captado a través de una ideación arraigada en la experiencia de cosas particulares. Patočka afirma que:

(...) el concepto de mundo no es una esencia general, porque solo hay un mundo, aquí la ideación [In-Idee-Setzen] no tiene sentido. Un mundo, en tanto que uno en principio, no es, sin embargo, un dato empírico, sino un dato a priori, ya que no se puede remontar ni a una experiencia individual ni a operaciones sobre ésta. El mundo no es una generalidad, sino un todo y sobre esta base es a priori (Patočka, 2000, p. 133).

El mundo, para Patočka, no es a priori en el sentido de una unidad fija, idealmente repetible, que puede ser captada a través de la multiplicidad de sus instanciaciones y cuya repetición está firmemente fundamentada en el doble acto subjetivo de repetición y captación de una esencia ideal a través de un espectro de particularidades. El mundo es una universalidad, pero no una que está fijada por un acto intencional que lo constituiría como una identidad, o como el polo idéntico de un acto intencional. El mundo, en resumen, no es un algo general llevado a la intuición por una conciencia ideadora (Patočka, 2000, p. 134).

Pero las cosas, o mejor la experiencia de un orden de cosas, como Husserl destacaría, no son irrelevantes para nuestra experiencia del todo. Más aún, sería un error considerar la orientación a la experiencia objetual como un mero prejuicio por parte de Husserl. La idea de que el todo del mundo primero llega a ser genuinamente significativo solo como un orden y ordenando las cosas, representa una importante e influyente convicción filosófica que encuentra una expresión poderosa en el pensamiento de Husserl. A su vez, lleva a Husserl a toda una serie de ideas, por las cuales es justificadamente famoso, con respecto a la naturaleza de la racionalidad científica, así como acerca de la tradición, la cultura y la historia de la razón en el más amplio de los sentidos. Al final, Patočka aprecia mucho más este aspecto del pensamiento husserliano, ${ }^{4}$ buscando, no tanto rechazarlo, sino encontrar formas de complementarlo, mostrando que el tema fenomenológico del mundo es más que la constitución de la naturaleza como un orden de las cosas. El

\footnotetext{
${ }^{4}$ Véase: Patočka (1998), pp. 106-107.
} 
mundo representa una universalidad que no es reductible a la razón, sino que, por el contrario, es intrínsecamente constitutiva del movimiento mismo de nuestras vidas como seres horizontales.

\section{EL CUERPO Y EL MOVIMIENTO}

Para comprender en qué sentido el mundo es algo más que el orden de las cosas se requiere, sin embargo, que consideremos cómo Patočka aborda el problema del cuerpo en la fenomenología. Las estructuras básicas de la orientación corporal, la actividad corporal, son para Patočka, no simplemente dadas "en" el mundo, sino que pertenecen de manera esencial a la manifestación del mundo mismo. La razón principal de esto es que el cuerpo, para Patočka, no solo se mueve, sino que es esencialmente movimiento, orientado por tres parámetros que a su vez definen su función en la manifestación del horizonte del mundo.

Dos de estos parámetros son desarrollados de la mano de los polos gemelos de la interioridad subjetiva y periférica mencionados anteriormente, pero deben tomarse en conjunto con un tercer parámetro que cristaliza el movimiento de la existencia humana como algo localizado corporalmente. Tomemos cada uno a su vez.

El primer parámetro interpreta el polo de la interioridad subjetiva como algo enrraizado, y esto en dos sentidos. Primero, el sujeto-cuerpo se relaciona con un referente inmóvil, lo que Patočka, en el ensayo de 1967 citado anteriormente, describe más bien poéticamente como la "tierra" (Patočka, 1967, p. 255). E1 vocabulario evoca aquí a Heidegger, pero la metáfora no es tan ajena a Husserl: porque la tierra, como también sostiene Husserl, en un sentido primordial no se mueve, es ese arché originario de la inmovilidad implícito en todo movimiento, y desde el cual el movimiento se proyecta a sí mismo. ${ }^{5}$ En segundo lugar, la tierra no solo es un referente, sino una fuerza tal que sostiene internamente la vida, y con esto se define la dependencia fundamental de la finitud, la existencia corporal (Patočka, 1967, p. 256).

El segundo parámetro constitutivo del cuerpo como movimiento es la distancia periférica que Patočka interpreta, nuevamente en lenguaje heideggeriano, con la metáfora del "cielo", que él describe como la distancia abierta que une al mundo como horizonte de sí mismo (Patočka, 1967, p. 256). Al igual que en Heidegger, el cielo está asociado con el movimiento de un comprender que proyecta posibilidades, abre el futuro y saca a la existencia subjetiva hacia afuera de sí misma.

El movimiento del cuerpo que se despliega entre la tierra y el cielo -o entre la necesidad intrínseca de enraizamiento existencial y el horizonte periférico del ser proyectado-, es descrito por Patočka como triangulado por un tercer parámetro, un

\footnotetext{
${ }^{5}$ Cf. Heidegger (2000), y Husserl (1934).
} 
centro primordial. Este centro concentra la actividad corporal en un movimiento de circunspección, uno tal que está fundado y trascendido por ambos, por la tierra y el cielo, respectivamente. El centro aquí no es un punto abstracto de referencia, sino una estructura existencial, cuyo componente más importante, argumenta Patočka, es una relación con el ser de otros, otros seres corporales que también existen en la región entre la tierra y el cielo. Y así, Patočka fija la estructura básica para un análisis de los movimientos de la existencia humana: "El contacto con otros es lo primordial, el más importante componente del centro del mundo natural, cuyo fundamento es la tierra y cuya periferia es el cielo." (Patočka, 1967, p. 258).

Ubicar a los otros en el centro del planteamiento es un giro importante en la comprensión de la espacialidad. Para Patočka, el movimiento de la actividad corporal humana es espacial en un sentido existencial precisamente porque la existencia humana, el ser personal, se relaciona consigo misma a través de lo otro, no accidentalmente, sino esencialmente. Este vínculo interno entre el espacio y lo otro es un tema clave en el planteameamiento patočkiano de los tres movimientos de la existencia humana, y se encuentra en la raíz de los fenómenos existenciales fundamentales relacionados con los dos primeros movimientos, como la vulnerabilidad, la necesidad de ser aceptado y la satisfacción cooperativa de las condiciones básicas de la vida (Patočka, 1998, pp. 148-151).

Vulnerabilidad y necesidad encarnan, sobre todo, primordialmente, la verdad básica de que la existencia humana, su sentido y su manera de ser en el mundo, siempre tiene lugar en una relación dialéctica con lo otro:

Nuestro cuerpo es una vida que es espacial en sí misma y de sí misma, produciendo su localización en el espacio y haciéndose espacial. El ser personal no es un sercosa, sino una auto-relación que, para actualizar esta relación, debe dar la vuelta a través de otro ser. Nos relacionamos con nosotros mismos al relacionarnos con lo otro, con más y más cosas y, en última instancia, con el universo como tal, para localizarnos en el mundo (Patočka, 1998, p. 31). ${ }^{6}$

Es importante enfatizar que, en la descripción de Patočka, estas relaciones con otros y con las cosas, gracias a las cuales encontramos (o mejor dicho, se produce) nuestro lugar en el mundo, tienen un carácter horizontal intrínseco, lo cual significa que involucran modalidades de distancia, ausencia, e indeterminación. Ahora bien, estas relaciones, aunque indeterminadas, no son completamente fluidas; ellas también tienen una densidad característica. Viviendo en horizontes, el ímpetu de la vida humana transfiere cierta actualidad a lo que todavía no es, a lo que está ausente o a lo que es indeterminado; las posibilidades mismas se condensan en el curso de una vida, extendiendo la realidad más allá de los confines estrechos de lo

\footnotetext{
${ }^{6}$ Cf. también Patočka (1998), p. 57.
} 
que está inequívocamente establecido y dado (Patočka, 1998, p. 36). Esto incluye posibilidades frente a las cosas, pero también hacia trayectorias de otras vidas, en la medida en que el todo de la existencia humana es el movimiento de ser lanzado hacia el exterior, de estar sujeto a un flujo centrífugo de cosas y de otros seres como nosotros mismos, todo lo cual nos obliga a alejarnos de nosotros mismos hacia el mundo. Los seres humanos son de esta manera seres de distancia, o mejor, seres que viven en una peculiar actualización las distancias:

Al vivir en los horizontes, la transferencia de actualidad apunta a una poderosa corriente centrífuga que gobierna nuestra vida, fuera de nosotros mismos, hacia el mundo. Vivimos alejados de nosotros mismos, siempre nos hemos trascendido en dirección al mundo, en dirección a sus regiones cada vez más remotas (Patočka, 1998, p. 36).

Este tema del movimiento podría decirse que pertenece a los inicios del pensamiento occidental, y de hecho, Patočka vincula explícitamente sus reflexiones sobre el movimiento de la existencia humana al concepto aristotélico de kinesis (Patočka, 1998, pp. 153-155). Al igual que Aristóteles, Patočka concibe el movimiento como el ser de la dunamis, el ser concreto de la posibilidad, la actualización de la potencia. No obstante, para Patočka el movimiento ya no depende, como sí para Aristóteles, de una actualidad existente a priori que serviría de fundamento; en lugar de eso, intenta una radicalización de la concepción aristotélica, hacia una afirmación existencial de que la existencia humana simplemente es un movimiento hacia abajo.

Sin embargo, al mismo tiempo el movimiento aquí es finito, condicionado; más aún, la ruptura con Aristóteles, en lo concerniente a la "actualidad" en cuanto condición previa, no es completa. El movimiento de la existencia humana, según Patočka, es lanzado en varias direcciones más allá de sí mismo, incluso hacia direcciones que en última instancia se refieren a una dependencia de lo que no se mueve, repitiendo así la figura de la necesidad de una "actualidad previa", al menos en un sentido ontológico amplio. Podría decirse que esto es parte del significado de las metáforas de la tierra y el cielo: representan polos constantes del movimiento de la vida humana en los horizontes, una solidez interna que corre a través de la apertura de lo posible. Tierra y cielo no son actualidades en el sentido aristotélico, ya que no son completos ni estáticos, pero están dados, a la manera de parámetros inmóviles constitutivos del horizonte del mundo. El horizonte del mundo denota, así, una apertura, en última instancia, la posibilidad en sí misma, pero también vincula, condiciona e incluso contiene. Esto significa que el movimiento humano, aunque autónomo, no está libre de fricción, densidad, lastre, esfuerzo, condicionamiento, incluso en alguna medida del auto-encierro y del acabamiento expresados por las cosas. 
Por encima de todo, el movimiento de la vida humana está ligado a la constante demanda de ser lanzado hacia sí mismo, por así decirlo, en el sentido de que la vida es lanzada hacia sí misma apuntando al horizonte de su propio no-estardada, el cual es, en muchos sentidos, más real que cualquier otra cosa que pueda contar inequívocamente como estando-dada en su actualidad. La antigua tesis de que la posibilidad es más alta que la actualidad, o que la realidad, encuentra asidero en el pensamiento de Patočka, pero al mismo tiempo se combina con la tesis de que el ser en posibilidades proyecta ante sí un prejuicio natural contra la positividad de lo actual, gracias al cual el horizonte del mundo se experimenta como algo real.

\section{INDIVIDUALIDAD Y REFLEXIÓN}

Esta descripción de una vida en posibilidades, en la que Patočka se esfuerza por captar la compleja relación de la vida con la otredad, así como por iluminar los patrones que conforman la concreción vivida o la densidad existencial de nuestra experiencia, es también el núcleo del enfoque de Patočka a propósito del problema de la individualidad. Esto, a su vez, implica abordar el problema del "yo" o el ego de la fenomenología clásica. Lejos de descuidar el tema del yo, un enfoque asubjetivo de la fenomenología está comprometido con una exploración de los múltiples sentidos del sí-mismo y de la personalidad que pertenecen a la proyección de la existencia humana en el mundo (Patočka, 1998, pp. 58-59).

Varias ideas importantes de Patočka encuentran su origen en este dominio de problemas. Quizás lo más importante gira en torno al problema del carácter histórico de la existencia humana, elaborado en sus famosos Ensayos Heréticos. Sin embargo, las reflexiones de Patočka también prometen reabrir un tema husserliano clave, el de la reflexión, en formas que tal vez no hayan sido plenamente apreciadas entre los comentaristas de Patočka, ni tal vez por el propio Patočka (Patočka, 1998, pp. 165-166). Este descuido es comprensible: en Husserl, la reflexión, la reflexión filosófica sobre todo, siempre parece estar demasiado en deuda con algún vestigio de la inmanencia cartesiana, ya sea en la forma del reiterado compromiso husserliano con el concepto de Brentano de "percepción interna", o con la influencia en su pensamiento de nociones relacionadas con la capacidad psicológica de un sujeto para volverse hacia adentro y capturarse en una mirada objetiva pura. Sin embargo, yo diría que hay una conexión más profunda entre los enfoques de Patočka y de Husserl sobre el problema que vale la pena considerar.

Tomemos, por ejemplo, la sugerencia de Patočka en sus conferencias de 1968/69 de que la reflexión debe entenderse como perteneciendo al campo de la apresentación, por lo tanto, como algo que se despliega en el contexto de nuestra relación con los demás (Patočka, 1998, p. 65). La idea es que la reflexión primitiva 
no es una forma de visión directa, sino que, al igual que la mirada de los otros, es decididamente una mirada apresentada, basada en la conciencia dinámica de ser mirado por otros. Patočka continúa argumentando que la mirada apresentada de uno mismo en su ser visto por otros conforma el contexto de un primer encuentro con uno mismo como un "sí-mismo". La re-flexión, en tanto que a-presentación, es, así, originaria: el sí-mismo no comienza completamente formado, sino que solo deviene reflejado a partir del sí-mismo de los otros; en otras palabras, como tal, el ser del sí-mismo primarimente deviene en el arco reflexivo inherente a la lógica del encuentro con los otros, gracias al cual el sí-mismo es introducido en la vida en un sentido primario.

El "sí mismo" es dado por otros, pero no en un sentido unívoco: lo dado aquí está determinado internamente por un proceso dialéctico, en el cual el movimiento originario de la existencia de uno forma el catalizador esencial, el fundamento insustituible y necesario para configurar el encuentro reflexivo en movimiento. El otro me da mi mí-mismo, pero solo en un movimiento que yo soy, y gracias al cual recibo este don de la individualidad absolutamente. Esto significa que, en el arco de reflexión, el ser que yo soy, el comprender que yo soy comienza desde sí mismo y se modifica efectivamente desde dentro: al salir de mí-mismo hacia otros, hacia el mundo, simultáneamente encuentro un retorno, una modificación de mi encuentro visual con el otro en un encuentro visual conmigo mismo.

Aquí es donde podemos identificar una conexión más profunda con la concepción husserliana de la reflexión, en el sentido de que podemos interpretar, creo yo, la concepción de la reflexión en Patočka como una re-articulación de una tesis importante de Husserl, que se encuentra en Ideas I y en otros lugares. Dicha tesis concibe la reflexión como una modificación básica de la conciencia (Husserl, 1976, §§77-78). Para Husserl, la reflexión representa un contramovimiento del símismo frente a su inmersión "natural" en las cosas, frente a esa fascinación por lo que es distinto de la conciencia, que oculta la presencia de la vida intencional para sí misma en favor de la manifestación de las cosas en el mundo. La reflexión es una modificación de la conciencia natural que resulta de una especie de inversión de su polaridad natural, una gracias a la cual la relación que guarda la conciencia con las cosas viene a articularse en sus propios términos, en lugar de permanecer en el prejuicio que permite que las cosas guíen el despliegue de la manifestación. En este sentido, la reflexión representa la modificación de un modo de mirar por otro, permitiendo la revelación de patrones de fenomenalidad que de otra manera solo operarían de forma anónima en la vida natural.

El enfoque de Patočka promete profundizar en esta explicación de la modificación como el origen de la reflexión, precisamente al proporcionar una concepción alternativa de la existencia "natural" de la conciencia presupuesta en la 
explicación de Husserl. Según Husserl, la modificación relevante para la reflexión tiene su origen en una capacidad innata de la conciencia natural: "original", la conciencia inmodificada alberga la capacidad de modular su forma, pasando de un modo de manifestación no-reflexivo a originar uno reflexivo. Con la reflexión situada en la esfera de la apresentación, por el contrario, Patočka insiste en una explicación mucho más compleja y dinámica de la modificación como el origen de la reflexión. Aquí, el "mirar" que emerge de la reflexión, el volverme hacia mí-mismo a lo largo de un camino transformado por el otro que me mira, pero sin reemplazarme, no está arraigado en el potencial interno de mi existencia para presentarme efectivamente a mí-mismo en una variedad de modos diferentes. La reflexión es aquí, de hecho, una modificación, incluso una transformación, pero no está basada en la libertad inmediata de la conciencia para dirigir el curso de sus modos de manifestación. Por el contrario, está arraigada en una condición original de la vida humana como estando en relación con lo no dado, o como estando abierta para que lo no-presente sature el terreno de la existencia, dando lugar a una forma única de auto-manifestación internamente determinada por la otredad. El sí-mismo "apresentado" que yo soy, ese sí-mismo que entra en mi vida por medio del canal interno de un retorno a través del otro, porta consigo la otredad del otro, esa otredad que a su vez constituye un parámetro original de lo que sale de mí-mismo en absoluto.

En otras palabras, la reflexión entendida como perteneciente a la apresentación propone un modo del mirar que tiene su origen en la otredad, en la no-presencia y en la horizontalidad de una vida originalmente vivida hacia fuera de sí misma. Patočka describe nuevamente la vida en términos del carácter existencial básico del movimiento, lo que significa que, en sentido amplio, la vida siempre es una modificación, sin ningún indicio de la presencia de un sí-mismo inalterado que haga las veces de su origen y fundamento.

\section{El PROBLEMA DE LA CLARIDAD}

Si el enfoque de Patočka en torno al problema de la reflexión está, al menos en cierta medida, en continuidad con la tesis básica de Husserl sobre su origen en la modificación, es igualmente cierto que rompe con la creencia husserliana de que la conciencia puede captar lo que es esencial por medio de un acceso al mundo a través de una reflexión objetivizante (Husserl, 1976, p. 162, §77). Patočka aquí apunta nada menos que a una nueva concepción de la claridad: "Si podemos mostrar una claridad que es en principio no-objetiva, y si todo lo objetivo, lo que objetiviza es la claridad, entonces todas las modalidades de la objetivización, están arraigadas en dicha claridad, y así nosotros hemos ido más allá de Husserl con la ayuda de un motivo husserliano" (Patočka, 1998, p. 84). 
Aunque Patočka no lo expresa en estos términos, el intento aquí equivale a demostrar que los límites esenciales de lo que puede contar como un objeto, articulado en el sentido más amplio posible, no pueden, a su vez, proporcionarnos una determinación clara de los límites de la claridad. El punto entonces sería que la claridad es más que objetividad, más que la posicionalidad de la creencia o los logros téticos del pensamiento en general.

Aquí, nuevamente, podemos observar que esto se basa en tendencias ya pertenecientes al propio desarrollo filosófico de Husserl. La reflexión fenomenológica en Ideas I se describe como un auto-compromiso viviente, una modalidad en la cual el flujo de la conciencia se manifiesta en y para sí misma; sin embargo, al mismo tiempo, la manifestación originaria de este flujo, que Husserl en otros lugares llega a describir como un "flujo heracliteo" (Husserl, 1950, p. 86), al principio cae fuera del alcance de la objetividad, incluso cuando ha sido afirmado en su darse originario (Husserl, 1976, §§67-71). La mera reflexión por sí sola no es una clarificación objetiva; debemos aprender a ver lo que está presente aquí, lo que la promesa de la reflexión hace visible, pero que aún no se encuentra plenamente realizado. El camino a seguir por Husserl es argumentar que, para que la conciencia se aclare, debe ponerse en evidencia, lo que a su vez debe tomar la forma de algún tipo de objetivación; de lo contrario, puede que se de "conciencia", pero permanecería encerrada en la oscuridad.

Patočka, en cambio, renuncia a lo que considera el prejuicio de que el acceso al ser solo se materializa, se completa, cuando se asienta en una forma de objetividad, ya sea empírica o eidética. En cambio, su convicción es que la existencia humana siempre tiene acceso a sí misma, que en su ser se mueve dentro de un tipo de claridad no-objetiva, aunque original. Aprendemos cómo explorar esta claridad de la existencia no a través de la evidencia, sino en términos de las posibilidades vividas que nos son dadas, ya que nuestra existencia solo es una apertura a lo que es, a lo que se manifiesta (Patočka, 1998, p. 101).

Algo más tiene que ver con esto, y nuevamente implica la tarea de confrontar al cartesianismo residual de la fenomenología clásica, sobre todo la tesis de que la auto-certeza del ego-cogito trae consigo una especie de auto-transparencia. Patočka se opone contundentemente a lo que considera un sesgo en la afirmación de esta tesis en la filosofía de Husserl:

¿Qué es la experiencia, la conciencia? Una claridad interpenetrada por la oscuridad. ¿Es realmente algo que puedo captar con total claridad en la visión interna, haciéndola totalmente transparente para sí misma? ¿O es la experiencia humana, por su propia naturaleza, algo esencialmente diferente de lo que puede darse en la experiencia objetiva? Esa es una pregunta que Husserl nunca planteó (Patočka, 1998, pp. 93-94). 
Y, nuevamente, Heidegger es fundamental para el propio intento patočkiano de plantear precisamente esa pregunta: "Heidegger pregunta cuál es nuestro acercamiento a nuestro propio ser. ¿Es una contemplación del ser? ¿Es una autoconciencia? ¿Es una mirada vuelta sobre mí mismo?” (Patočka, 1998, p. 95).

Sin embargo, la respuesta de Patočka a esta pregunta es decididamente "sí y no"; una ambivalencia señalada más adelante en la misma conferencia: "Una mirada interior es, naturalmente, también una parte de lo que somos, pero nuestro propio ser tiene un acceso más directo a sí mismo" (Patočka, 1998, p. 95). Esta declaración se hace en el contexto de la discusión patočkiana de lo que él considera la orientación práctica de la filosofía de Heidegger, al menos como se había articulado en la primera división de Sein und Zeit. En cuanto acción proyectada, la comprensión que el Dasein tiene de sí mismo es, en un sentido fundamental, "no-objetivante", en el sentido de que el alcance de su auto-comprensión no se limita al dominio de la autorepresentación objetiva. Este reconocimiento de la importancia de las dimensiones prácticas y no objetivantes de la vida humana, junto con el tema de la tendencia a la auto-obstrucción o caída, representan para Patočka "las grandes ventajas de Heidegger sobre la fenomenología husserliana" (Patočka, 1998, p. 97).

Sin embargo, debemos tener en cuenta que, en el pensamiento de Patočka, el énfasis no radica tanto en la primacía de lo práctico per se, sino en el carácter personal que caracteriza la acción humana como una manera de ser. O mejor, el énfasis no está en el hecho de que los seres humanos actúen, de que sus vidas estén ocupadas en asuntos prácticos, sino en que actúen desde una no-indiferencia hacia símismos, hacia lo que está en juego en la existencia de uno. Analizada por Heidegger bajo el título de Sorge (cuidado), esta preocupación por uno mismo, que determina el horizonte de un quehacer existencial, es tanto una realización del sentido como la ejecución exitosa de una práctica u otra (Patočka, 1998, p. 97). Esto significa que, como lo era también para Heidegger, el enfoque patockiano no consiste en favorecer la esfera práctica sobre la teórica, sino en argumentar que hay algo común, una raíz existencial afín a ambos aspectos en la estructura-del-cuidado del Dasein.

El resultado es que el tema heideggereano de la propiedad (Eigenheit) es posiblemente más importante para Patočka que cualquier tipo de pragmatismo que uno podría tener la tentación de inferir desde Sein und Zeit. El análisis de la propiedad constituye la base de la crítica patočkiana a las concepciones egocéntricas o de una "yoidad" que ignoraría esta característica existencial básica en favor de un concepto del yo como fundamento o primer constructo, por el cual lo que está en juego en la propiedad se convierte en una especie de principio estructural de composición (Heidegger, 1927, §§39-42). Tales concepciones "fundacionales" interpretan efectivamente el "yo" en términos de una indiferencia básica hacia el sí-mismo: el ser del cuidado, el vivir de la vida como la tarea de ser, 
es sustituido por un principio anónimo de organización. Contra esto, y siguiendo a Heidegger, Patočka argumenta que "debemos comprender el yo en el contexto de ese ser que se relaciona con su propio ser, al que su ser no le es indiferente, el yo como una tarea, el ser como una tarea, siendo la cual se acepta y se realiza a sí mismo." (Patočka, 1998, p. 100). El yo, en otras palabras, no es para Patočka un fundamentum, un garante de orden estructural-arquitectónico, sino que es un spatium, definido por la propiedad, o por el juego concreto de las maneras en que los humanos están abiertos a sí mismos.

\section{EL PRINCIPIO DE TODOS LOS PRINCIPIOS}

Tomados en conjunto, los elementos de una fenomenología a-subjetiva que hemos estado considerando, a saber: el análisis del horizonte del mundo, el concepto de existencia humana como movimiento corporalizado, la ubicación de una teoría de la reflexión en la apresentación, la crítica al yo y la recuperación del tema de la propiedad, pueden tomarse como un impulso hacia una revisión fundamental del famoso "principio de todos los principios" de Husserl, tal y como es expuesto en la sección 24 de Ideas I (Husserl, 1976, p. 51). La tendencia general del enfoque de Patočka, podríamos argumentar, es interrumpir una lectura de ese principio que privilegiaría la donación en el sentido de una auto-donación, o Selbstgegebenheit, sobre la donación en el sentido de la donación originaria, u originär Gegebenheit.

Estos dos sentidos de la donación, por supuesto, generalmente trabajan juntos en Ideas I, y específicamente en la elaboración metodológica de Husserl en el $\S 67$, en el que los términos utilizados en el principio de todos los principios encuentran su primera determinación. En esta última sección, Husserl argumenta una vez más que la intuitividad de la donación originaria (originär gebende Anschauung) proporciona el espacio, el horizonte en el que algo se manifiesta "como" sí mismo (Selbst), por lo tanto, como auto-dado. Pero lo uno no necesariamente coincide con lo otro: solo la auto-donación en última instancia proporciona un ejemplo de claridad, la cual puede decirse que produce el conocimiento. Husserl, por lo tanto, tiende a enfatizar la donación consumada de algo como sí mismo, gracias a las dinámicas constitutivas de la vida intencional que se alimentan de las potencialidades de la donación originaria para llevar las cosas a la claridad de la auto-donación. Esto implica que la donación originaria no tiene ninguna reivindicación que sea independiente de la orientación intuitiva, tampoco del conocimiento, aparte de su rol como una especie de iniciación, o como un terreno preparatorio para el cumplimiento de la auto-donación (Husserl, 1976, p. 142). ${ }^{7}$

\footnotetext{
${ }^{7}$ Véase: Patočka (2000), pp. 135-138.
} 
Aquí, nuevamente, la reflexión, o al menos una reflexión que se conformaría en una objetivización, en una auto-presencia de la donación, se encuentra bajo una presión crítica en el compromiso de Patočka con Husserl, especialmente cuando el sujeto en consideración es uno-mismo. Es decir, la pregunta es si esta trayectoria desde lo originario hasta la auto-presencia, al rastrearla de cerca, produce una captación genuinamente "original” del sí-mismo:

La auto-presencia de algo en su origen todavía no garantiza que se de en su modo originario. El criterio de Husserl de la verdad filosófica, el requisito de la responsabilidad basada en la reflexión, no es suficiente. Es verdad, en la reflexión, en cierto sentido, yo soy dado a mí-mismo, pero ¿éste es un yo en el sentido más original posible? ¿Es la reflexión en el sentido de auto-objetivación la puerta de entrada a mí mismo? ¿No debemos ir más allá, extendiendo el concepto de reflexión para incluir también ese procedimiento filosófico que capta no solo lo que se da, sino también la implicación interna del sentido de lo dado, que apunta más allá? (Patočka, 1998, p. 102).

Aquí nada menos que el movimiento hacia la auto-donación en sí mismo se vuelve sospechoso, ya que posiblemente implica una trama que oscurece la dinámica existencial en la cual el yo se hace presente para la reflexión, dándose allí como "lo mismo", pero que en realidad es quizás, en el mejor de los casos, solo un derivado, incluso una auto-presentación ilusoria. La importancia escéptica de esta cuestión se puede generalizar para incluir una crítica a la concepción husserliana de la reflexión filosófica, basada en su principio de todos los principios. Esta crítica implica que el principio metodológico que moldea la reflexión está diseñado de tal manera que se cierra al compromiso que la auto-donación tiene con la experiencia subjetiva, amenazando con oscurecer la "verdad" más primordial de esa experiencia en sí misma. Al convertirse en observador imparcial de la vida intencional, la reflexión deja de lado el cumplimiento de la auto-donación que la vida ha elegido para su manifestación; al pretender estar libre de cualquier pre-juicio teórico que predetermine lo que se supone que es, la reflexión corre el riesgo de sucumbir en una ingenuidad básica con respecto a la naturaleza de lo dado en cuanto existencia subjetiva. ${ }^{8}$

Más aún, esta inocente ingenuidad del mero observador corre el riesgo de oscurecer el sentido existencial de la reflexión misma. La reflexión, argumenta Patočka, está fundada en la apertura de ese acceso original de los seres humanos a su propio ser, en el sentido en que la existencia misma está no-cerrada para sí misma, precisamente en el modo de la no-indiferencia. Y aquí Patočka quiere aludir, precisamente, a la no-indiferencia de un ser que debe buscar-se afuera de sí mismo,

\footnotetext{
${ }^{8}$ Véase: Husserl (1950), §15, y también: Patočka (2000), pp. 227-230.
} 
que es no-indiferente ante todo porque es originalmente un problema para sí mismo (Patočka, 1998, p. 111). Aquí está el desafío para Husserl: Patočka propone la idea de una reflexión nada más que como el movimiento hacia adelante de la vida problemática, una que da forma y produce un "yo", un sí-mismo, como un proyecto, en contraste con la concepción husserliana de una reflexión que se esfuerza por ser "desinteresada", en el sentido en que busca no dar forma ni modificar esencialmente $n a d a$, intentando ser un medio perfectamente transparente en el que la vida se manifieste inequívocamente para sí misma.

Uno debe tener precaución aquí, y no exagerar las cosas. La vida, para Husserl, está saturada de intereses y motivaciones, que pertenecen a su propia esencia; el punto no fue nunca recortar la reflexión a partir de los intereses de la vida, sino moldear la reflexión de tal manera que proporcionara un espacio neutral que permitiera que las complejidades de la vida interesada se manifestaran, en cualquiera de las formas en las que se da cumplimiento a sí misma en su ser. Es solo cuando consideramos la caracterización husserliana de este modo de reflexión como algo "absoluto", arraigado en la donación originaria de una experiencia igualmente absoluta que, en última instancia, proporciona forma y dirección a toda manifestación, cuando el significado de la alternativa de Patočka se vuelve claro. La reflexión, para Patočka, en contraste con la observación, aunque se la conciba adecuadamente, no deja de ser "una parte de la vida humana en el mundo, un momento de la vida humana auténtica", lo que significa que en última instancia no puede ser neutral, no puede abstenerse de la tarea de lidiar con las tendencias de auto-descubrimiento, auto-pérdida y auto-ofuscación que pertenecen al movimiento mismo de la existencia humana (Patočka, 1998, p. 119). Lo problemático del absolutismo husserliano es, para Patočka, precisamente el aparente intento de suspender la parcialidad de la vida como un presupuesto para la articulación de la fenomenalidad del fenómeno, como si la imparcialidad garantizara una perspectiva (incluso una perspectiva "científica") sobre el drama de la manifestación. O como si la fenomenalidad, el secreto del aparecer, fuera algo intrínsecamente neutral, y accesible en su completud solo a través de una postura subjetiva que ha logrado asegurar su propia neutralidad proporcional. La objeción de Patočka surge de su intuición de que existe un límite básico para cualquier ejercicio de neutralidad en los asuntos filosóficos, es más: que el vínculo interno entre la existencia humana y la manifestación podría ser de alguna manera algo "neutral". Esta objeción está dirigida directamente en contra de la convicción del último Husserl, argumentada, debe decirse, con una vehemencia y una penetración análogas a las de Patočka, de que tal neutralidad es esencial para la posibilidad misma de la autonomía filosófica, gracias a la cual el campo de los fenómenos, y junto con éste la vida en sí misma, puede ser auténticamente articulado por el pensamiento. 


\title{
LA ANIMALIDAD COMO TEMA FILOSÓFICO
}

Un aspecto final de la fenomenología asubjetiva de Patočka que quisiera resaltar es su compromiso con los importantes fenómenos de la afectividad y la sensibilidad, y su relación con otros posibles modos de ser distintos del humano que evidentemente están contenidos en lo humano:

\begin{abstract}
Sigue siendo un problema sin resolver cómo podríamos rastrear, cómo podríamos delimitar ontológicamente la afectividad y la emotividad en algo que es meramente viviente, que carece de existencia. Sin embargo, tenemos que lidiar con eso porque nuestra existencia humana en un mundo (funcional y pragmático) presupone la existencia de lo infantil y lo animal en nuestro interior (Patočka, 1998, p. 138).
\end{abstract}

Yo diría que esta línea de cuestionamiento tiene el potencial de abrir una perspectiva fenomenológica sobre el problema de la animalidad humana. Aquí también se podría decir que tanto Husserl como Heidegger, en sus reflexiones sobre el tiempo, ya anticipan la dirección general en la que podría desarrollarse el problema. Para ambos, el comprender humano toma la forma de un punto de partida, y con eso se abre una especie de distancia frente a lo que pertenece a los reinos de la sensibilidad y la afectividad. Esta distancia del comprender se fundamenta en su interpretación del tiempo como pasaje, que toma la forma general de un contraste entre algo cerrado (el pasado) y algo abierto (el futuro): el comprender opera en esa oscilación entre un pasado absorbido en su clausura y un futuro que se abre a la clausura del pasado, pero sin estar separado de él.

Podemos ver que el pasaje del tiempo sugiere, ciertamente como un aspecto implícito de su dinámica, una relación potencial con el mundo que todavía no está marcada por la distancia total del comprender, donde la oscilación entre la clausura del pasado y la apertura del futuro aún no se ha desenvuelto como tal. El tiempo mismo sugiere la posibilidad de un ser que aún no es un movimiento entendido como la plena realización del pasaje del tiempo, sino que deja un tiempo no-realizado como el fundamento potencial para el establecimiento de una vida en distancias y, con todo, sigue siendo una persistencia en la proximidad (Patočka, 1998, p. 138).

Esta dimensión de la existencia que todavía no es existencia, esta “animalidad" para darle otro nombre, está representada en la descripción patočkiana de los movimientos de la existencia humana como una dimensión disruptiva y reprimida (Patočka, 1998, pp. 158-159). Sin embargo, al mismo tiempo tiene un rol positivo, ya que proporciona a la vida un sentido de profundidad, y con eso un sentido de totalidad cósmica intrínseca al significado 
del mundo que nunca puede incorporarse sin ambigüedades en una perspectiva que siempre lo haya dejado atrás.

Este potencial para un análisis de la animalidad humana es, creo yo, una de las promesas más heréticas de la fenomenología asubjetiva de Patočka, una que se aleja de la persistente prohibición de la fenomenología clásica contra la antropología filosófica. ${ }^{9}$ Patočka, uno podría argumentar, ni afirma la tesis de una vida que funciona de forma anónima, como una especie de ser absoluto de distancia irrevocable (y con un cumplimiento temporal) que formaría el horizonte definitivo de toda experiencia, ni sigue el giro heideggeriano que aparentemente descentraría la autocomprensión del Dasein en favor de un destino igualmente anónimo del ser. En cambio, inspirado por Eugen Fink, Patočka busca revivir la posibilidad de un sentido más antiguo del mundo como el acontecimiento de la manifestación, como un gran misterio irreductible a la subjetividad y a su dinámica de experiencia y comprensión, si bien está implícito en todo:

Es una condición para la aparición de un existente que esté en un lugar, en un tiempo, siempre dentro de una cierta comprensión del tiempo y el espacio. ¿No existe entonces en nosotros cierta comprensión, poco clara, anticipatoria, noobjetivable, de esa totalidad que antecede? ¿No podríamos acoger, así, en un contexto ontológico, lo que crea la luz para aparecer, la totalidad de todo lo que es, en el sentido fuerte de la palabra? ¿No sería esto una rehabilitación de la antigua idea de physis como arche? (Patočka, 1998, p. 169).

Physis, cuyo profundo sentido es transmitido por la animalidad hacia el nucleo de la afectividad, nos remite al tema del cuerpo, aunque ahora de manera considerablemente más profunda gracias a la noción patočkiana de una vida disruptiva en la proximidad. Considero que el gesto básico de Patočka aquí es el intento de enmarcar el problema de la individuación en términos de este sentido de un origen oculto que permanece eternamente indiferente a sí mismo, un sentido que se encuentra, tal vez paradójicamente, en el corazón de la no-indiferencia de la existencia corporal. El cuerpo, considerado como un movimiento, está situado en una línea divisoria primordial entre un todo eternamente indiferente a sí mismo, y el movimiento de una existencia que es esencialmente no-indiferente, un problema para sí misma y una búsqueda de sí misma. La fenomenalidad del cuerpo está así marcada en la dinámica entre una insuperable resistencia cósmica a aparecer, y un constante esfuerzo por ver, por dejar que aparezca. Esta tensión es lo que, en mi opinión, al final representa para Patočka el origen formativo del movimiento productivo de la manifestación. Y también es el cuerpo el que asegura así que cualquier experiencia del sí-mismo, cualquier búsqueda de uno-mismo, involucre

\footnotetext{
${ }^{9}$ Véase: Husserl (1989), pp. 164-181; Heidegger (1927), §10.
} 
ambas dimensiones aparentemente irreconciliables como aspectos del ser en el mundo, porque su intermedio no es más que el vínculo mismo entre la individuación corporal y el horizonte del mundo como tal.

\section{CONCLUSIÓN}

La trayectoria general del pensamiento de Patočka es, por supuesto, mucho más amplia que lo que podría sugerir el proyecto específico de una fenomenología asubjetiva, aunque espero haber indicado al menos cuán importante permanece este último en cualquier intento de comprender la totalidad del trabajo filosófico de Patočka. Los temas del sentido, del mundo, del cuerpo, del movimiento, de la individualidad y de la reflexión están tan relacionados con el pensamiento político y la filosofía de la historia de Patočka, como lo están con sus lecturas de la tradición fenomenológica de Husserl y Heidegger, incluso si las ideas que se encuentran en aquél no son estrictamente rastreables en los esfuerzos de éste. Más aún, el surgimiento de los temas de la animalidad y la physis en la filosofía posterior de Patočka se debe en gran medida al proyecto de una fenomenología asubjetiva, e indican que su promesa es la base para una sorprendente renovación de la reflexión ontológica sobre el ser del mundo.

En el espíritu de una reflexión sobre la promesa del pensamiento patočkiano, le dejaré la última palabra, citando un pasaje que creo que captura el alcance completo de la visión filosófica que lo guió en su búsqueda de una fenomenología asubjetiva, y que podría servir perfectamente como su coda:

Es como corporales que somos individuos. En su corporeidad, los seres humanos se encuentran en el borde entre ser indiferentes a sí mismos y a todo lo demás, y la existencia en el sentido de una relación pura con la totalidad de lo que es. Sobre la base de su corporeidad, los humanos no son solamente seres de distancia, sino también seres de proximidad, seres enraizados; no solo seres intramundanos sino también seres en el mundo (Patočka, 1998, p. 178). 
Heidegger, Martin (1927). Sein und Zeit. En Herrmann, Friedrich-Wilhelm (ed.) (1977). Gesamtausgabe de Heidegger, vol. 2. Frankfurt: Klostermann.

Heidegger, Martin (2000) “Bauen Wohnen Denken”. En Herrmann, Friedrich-Wilhelm (ed.). Vorträge und Aufsätze. Gesamtausgabe 7. Frankfurt am Main: Klostermann, pp. 145-164.

Husserl, Edmund (1934). "Kopernikanische Umwendung der Kopernikanischen Umwendung“. En Dünne, Jörg y Günzel, Stephan (eds.) (2006). Raumtheorie. Grundlagentexte aus Philosophie und Kulturwissenschaften. Frankfurt am Main: Suhrkamp, pp. 153-164.

Husserl, Edmund (1950). Cartesianische Meditationen und Pariser Vorträge, Husserliana I. Strasser, Stephan (ed.) The Hague: Nijhoff.

Husserl, Edmund (1976). Ideen zu einer reiner Phänomenologie, Husserliana III. Schuhmann, Karl (ed.). The Hague: Nijhoff.

Husserl, Edmund (1989). "Phänomenologie und Anthropologie“. En Nenon, Thomas y Sepp, Hans Rainer (eds.). Aufsatze und Vorträge (1922-1937). Husserliana, XXVII.

Dordrecht: Kluwer, pp. 164-181.

Patočka, Jan (1967). “The 'Natural' World and Phenomenology”. En Kohák, Erazim (ed.) (1989). Jan Patočka. Philosophy and Selected Writings. Chicago: University of Chicago Press, pp. 239-273.

Patočka, Jan (1969). “Co je existence?”. Filosofický časopis 17/5-6, pp. 682-702.

Patočka, Jan (1970). "Der Subjektivismus der Husserlschen und die Möglichkeit einer ,asubjektiven' Phänomenologie”. En Die Bewegung der menschlichen Existenz. Phänomenologische Schriften II, Jan Patocka: Ausgewählte Schriften. Nellen, Klaus, Němec, Jiří, Srubar, Ilja (eds.). Stuttgart: Klett-Cotta, 267-309.

Patočka, Jan (1971). "Der Subjektivismus der Husserlschen und die Forderung einer asubjektiven Phänomenologie". En Die Bewegung der menschlichen Existenz. Phänomenologische Schriften II, Jan Patocka: Ausgewählte Schriften, Nellen, Klaus, Němec, Jiř́i, Srubar, Ilja (eds.). Stuttgart: Klett-Cotta, 317-334.

Patočka, Jan (1972). "Edmund Husserl's Philosophy of the Crisis of the Sciences and His Conception of a Phenomenology of the 'Life-World'. Kohák, Erazim (ed.) (1989). Jan Patočka. Philosophy and Selected Writings. Chicago: University of Chicago Press, pp. 223-238.

Patočka, Jan (1998). Body, Community, Language, World. Kohák, Erazim (trad.). Chicago (II): Open Court.

Patočka, Jan (2000). Vom Erscheinen als solchem. Texte aus dem Nachlaß, Text Nr. V. Blaschek-Hahn, Elga y Novotný, Karel (eds.). Freiburg/München: Karl Alber. 